\title{
Special issue on natural and secure computing in modern soft computing application
}

\author{
Ilsun You ${ }^{1} \cdot$ Marek R. Ogiela $^{2} \cdot$ Fang-Yie Leu $^{3}$
}

Published online: 28 March 2016

(C) Springer-Verlag Berlin Heidelberg 2016

The rapid development of modern computer and Internet technologies is strongly connected with creation of the new computational paradigms as well as advanced information systems for secure communication between smart tech devices and humans. One of the reasons for the big progress in modern computer and communication technologies is due to the state-of-the-art achievements in soft computing approaches, cognitive computer science and a great number of other algorithmic techniques. It is also inspired by biology solutions (artificial intelligence, bioinspired computation, etc.). Recently, such methods have had a great importance. We can utilize these methods to intelligently analyze a great amount of complex data as well as manage in secure manner the data transmissions over global communication networks. The possibility of further development of such technology will depend on many factors, such as designing new solution in security areas, and introducing a generation and cognitive information systems based on natural computation approaches. These subjects, as well as a number of others, like the ways of using biological models to invent new solutions in the field of natural computing, computational intelligence and secure communication pro-

$凶$ Ilsun You

ilsunu@gmail.com

Marek R. Ogiela

mogiela@agh.edu.pl

Fang-Yie Leu

leufy@thu.edu.tw

1 Department of Information Security Engineering, Soonchunhyang University, Asan, Korea

2 AGH University of Science and Technology, Kraków, Poland

3 Tunghai University, Taichung, Taiwan tocols, will form the subject of a special issue on Natural and Secure Computing in Modern Soft Computing Application.

This time, our call for papers was warmly responded from all over the world. After rigorous review, only 13 papers were accepted. They provide readers with a showcase of the most recent challenges and advances in the topics of this special issue. We believe that these papers can indeed trigger further R\&D improvements as well as promote state-of-theart projects and case analyses of practical significance related to this important subject. In the following, we will briefly introduce these papers one by one.

The first paper entitled Data Stream Visualization Framework for Smart Cities by Villanueva et al. (2015) considers that monitoring smart cities is a key challenge due to the variety of data streams generated by different processes (traffic, human dynamics, pollution, energy supply, water supply, etc.). All these streams show what, where and when something is happening in the city. This paper applied different types of glyphs for illustrating real-time stream evolution of data gathered in the city. The purpose is making the most out of human capability for outperforming in detecting visual patterns.

The second paper, entitled Using Fuzzy Logic to Reduce Ping-Pong Handover Effects in LTE Networks by Tsai et al. (2015) proposed a fast and simple fuzzy-logic-based handover decision system, named fuzzy-based low ping-pong effect handover system (FPEHS), to reduce the ping-pong effect in an LTE network. In the FPEHS, five parameters, including current signal-to-noise ratio (SNR), detected SNR, bandwidth of serving eNB, bandwidth of target eNB, and remaining energy of the underlying user's device, are input to the fuzzy logic unit to make handover decision. Their simulation results show that the FPEHS can effectively decrease the ping-pong effect to about $92.94 \%$, on average compared with that of the standard LTE's handover mechanism. 
Further, people in their workplaces often neither receive energy consumption feedback nor do they pay a monthly invoice to electricity providers. To enhance workers' energy awareness, the third paper named Embedding Intelligent Eco-aware Systems within Everyday Things to Increase People's Energy Awareness by Casado-Mansilla et al. (2015) transformed information of everyday-shared electrical appliances, such as beamers, coffee makers, printers, screens, portable fans, kettles, and so on, into persuasive eco-aware everyday things. In fact, the information is transformed into time series and then processed to obtain the appliances' next-week usage forecast. Autoregressive Integrated Moving Average model has been selected as the potentially most accurate method after comparing with three different configurations of artificial neural networks.

In the fourth paper, entitled Image Processing Acceleration for Intelligent Unmanned Aerial Vehicle on Mobile GPU by Jeon et al. (2015), presented an algorithm for providing vision-guided unmanned aerial vehicle (UAV) control using vision information that is processed on a mobile graphic processing unit (GPU). Most real-time machine vision applications for UAVs exploit low-resolution images because of the shortage of computational resources. However, GPUs quickly emerged as inexpensive parallel processors providing high computation power in mobile environment. This paper presents an approach for detecting and tracking lines that use a mobile GPU. Hough transform and clustering techniques were used for robust and fast tracking.

The fifth paper named A Trusted User-to-Role and Roleto-Key Access Control Scheme by Chen (2015) is the first that proposed the TURA role-based access control (TURARBAC) scheme, which as a new trusted role-based access control (RBAC) model, can give a solution to cope with the problem on when many malicious users who want to launch insider threats (InTs) will be assigned to an RBAC system. In other words, the untrusted case is a type of InTs. The scheme takes the advantages via soft computing approaches chosen by the system to evaluate each user in an interactive session. Upon finding some malicious access contents, the user's role for accessing the system and the role-based RSA key are both deleted.

Currently, existing dummy-based location privacy protection schemes, like various location-based services (LBSs) and others, cloud systems cannot provide long-term privacy protection. For this, the sixth paper named Long-term Location Privacy Protection for Location-based Services in Mobile Cloud Computing by Tang (2015) claimed four principles for the dummy-based long-term location privacy protection (LT-LPP). Based on the principles, a set of longterm consistent dummy generation algorithms for the LT-LPP is proposed, which uses soft computing techniques to balance the preferred privacy protection and computing cost. The experimental results demonstrate that this approach is effective to both long-term privacy protection and fake path generation for LBSs in mobile clouds.

The seventh paper called Big Data: The Key to Energy Efficiency in Smart Buildings by Victoria Moreno et al. (2015) presented a novel approach for energy saving in buildings through the identification of the relevant parameters and the application of soft computing techniques to generate predictive models of energy consumption in buildings. With these models, it is possible to define strategies for optimizing the day-to- day energy consumption in buildings. To verify the feasibility of this approach, the authors applied it to a referenced building from which monitoring contextual data for a complete year can be obtained.

The eighth paper entitled TACIoT: Multidimensional Trust-aware Access Control System for the Internet of Things by Bernabe et al. (2015) proposed a flexible trust-aware access control system for IoT (TACIoT), which provides an end-to-end and reliable security mechanism for IoT devices, based on a lightweight authorization mechanism and a novel trust model that has been specially devised for IoT environments. TACIoT extends traditional access control systems by taking into account trust values which are based on reputation, quality of service, security considerations and devices' social relationships.

The ninth paper named Random-Oracle Based Anonymous Credential System for Efficient Attributes Proof on Smart Devices by Guo et al. (2015) proposed two extended signature schemes, BLS+ and BGLS+, to be cryptographical building blocks for constructing anonymous credentials in the random oracle model. Identity-like information of message holder is encoded in a signature so that the message holder can prove the possession of the input message along with the validity of a signature. This paper also presented an issuance protocol for anonymous credentials embedding weak attributes which are referred to what cannot identify a user in a population. Users can prove any combination of attributes all at once by aggregating the corresponding individual credentials into one. The attributes proof protocols on AND and OR relation over multiple attributes are also given.

The tenth paper named Multilevel Threshold Selection for Image Segmentation using Soft Computing Techniques by Mala and Sridevi (2015) considered that multilevel thresholding is a method which can be applied to segment a given image into unique sub-regions when gray value distribution of the pixels is not distinct. The segmentation results are affected by factors such as number of threshold and threshold values. Hence, this paper proposed different methods for determining optimal thresholds using optimization techniques, namely GA, PSO and hybrid model. Parallel algorithms are also proposed and implemented for these methods to reduce the execution time. From the experimental results, it is inferred that the proposed methods take less 
time for determining the optimal thresholds compared with existing methods such as Otsu and Kapur methods.

The 11th one named Recognizing Multiple Emotion from Ambiguous Facial Expressions on Mobile Platforms by Lee et al. (2015) introduced a multiple emotion recognition system that can recognize combinations of up to a maximum of three different emotions using an active appearance model (AAM), the proposed classification standard, and a k-nearest neighbor ( $\mathrm{k}-\mathrm{NN}$ ) classifier in mobile environments. AAM takes the expression of variations calculated by the proposed classification standard according to changes in human expressions in real time. The proposed k-NN classifies basic emotions (normal, happy, sad, angry, surprise) as well as more ambiguous emotions by combining the basic emotions also in real time, and each recognized emotion that can be subdivided has its own strength.

The 12th paper proposed, An Effective and Intelligent Windows Application Filtering System using Software Similarity by Kim et al. (2015), designed and implemented an efficient and intelligent software filtering system based on software similarity. This system measures similarity of the characteristics extracted from an original program and a suspicious one (or a cracked one), and then determines whether the suspicious program is a cracked version of the copyrighted original program based on the similarity measure.

The last one entitled Security System Architecture for Data Integrity based on a Virtual Smart Meter Overlay in a Smart Grid System by Lim et al. (2015) proposed an architecture of a security system to provide secure and reliable smart grid service, including a data authentication process to verify the aggregated data and virtual network management to detect a compromised area or node. It detects a false data injection attack and further prevents a denial of service (DoS) attack efficiently with less overhead for the individual devices comprising the smart grid system.

After the introduction, now we would like to say that after going through a rigorous peer review procedure and revision following independent reviewers' comments, the 13 papers presented in this special issue make a significant contribution to academic researchers, industry professionals, students, and all readers interested in this subject, working or wanting to extend their knowledge from the areas of Natural and Secure Computing in Modern Soft Computing, as well as privacy amplification, particularly for securing Internet services, cloud security, and cryptography.
We would also like to express our sincere appreciation to all the authors for their valuable contributions. Our special thanks go to the editorial board for this special issue and Professor Antonio Di Nola, Editors-in-Chief of Journal of Soft Computing, for the invitation to organize this special issue and the great support throughout the entire publication processes.

\section{Compliance with ethical standards}

Conflict of interest The authors declare that they have no conflict of interest.

\section{References}

Bernabe JB, Ramos JLH, Gomez AFS (2015) TACIoT: multidimensional trust-aware access control system for the internet of things. Soft Comput. doi:10.1007/s00500-015-1705-6

Casado-Mansilla D, Lopez-de-Armentia J, Venturay D, Garaizar P, Lopez-de-Ipina D (2015) Embedding intelligent eco-aware systems within everyday things to increase people's energy awareness. Soft Comput. doi:10.1007/s00500-015-1751-0

Chen H-C (2015) A trusted user-to-role and role-to-key access control scheme. Soft Comput. doi:10.1007/s00500-015-1715-4

Guo N, Gao T, Park H (2015) Random oracle-based anonymous credential system for efficient attributes proof on smart devices. Soft Comput. doi:10.1007/s00500-015-1704-7

Jeon D, Kim D-H, Ha Y-G, Tyan V (2015) Image processing acceleration for intelligent unmanned aerial vehicle on mobile GPU. Soft Comput. doi:10.1007/s00500-015-1656-y

Kim D, Kim Y, Cho S, Park M, Han S, Lee G, Hwang Y (2015) An effective and intelligent windows application filtering system using software similarity. Soft Comput. doi:10.1007/s00500-015-1678-5

Lee Y-H, Han W, Kim Y (2015) Recognizing multiple emotion from ambiguous facial expressions on mobile platforms. Soft Comput. doi:10.1007/s00500-015-1680-y

Lim J, Doh I, Chae K (2015) Security system architecture for data integrity based on a virtual smart meter overlay in a smart grid system. Soft Comput. doi:10.1007/s00500-015-1864-5

Mala C, Sridevi M (2015) Multilevel threshold selection for image segmentation using soft computing techniques. Soft Comput. doi:10. $1007 /$ s00500-015-1677-6

Tang F, Li J, You I, Guo M (2015) Long-term location privacy protection for location-based services in mobile cloud computing. Soft Comput. doi:10.1007/s00500-015-1703-8

Tsai K-L, Liu H-Y, Liu Y-W (2015) Using fuzzy logic to reduce pingpong handover effects in LTE networks. Soft Comput. doi:10. 1007/s00500-015-1655-z

Victoria Moreno M, Dufour L, Skarmeta AF, Jara AJ, Genoud D, Ladevie B, Bezian J-J (2015) Big data: the key to energy efficiency in smart buildings. Soft Comput. doi:10.1007/s00500-015-1679-4

Villanueva FJ, Aguirre C, Rubio AB, Villa D, Santomfiia MJ, Lopez JC (2015) Data stream visualization framework for smart cities. Soft Comput. doi:10.1007/s00500-015-1829-8 